\section{REDUCTION OF MULTIPLE-ACCESS INTERFERENCE FOR OPTICAL CDMA SYSTEMS}

\author{
Maw-Yang Liu ${ }^{1}$ and Hen-Wai Tsao ${ }^{1}$ \\ ${ }^{1}$ Department of Electrical Engineering \\ and Graduate Institute of Communication Engineering \\ National Taiwan University \\ Taipei, Taiwan 107, R.O.C.
}

\section{Received 18 January 2001}

\begin{abstract}
Interference reduction via a cancellation scheme for synchronous optical CDMA systems is discussed. Based on integrating over one bit duration of the received optical signal, the interfering signals can be estimated and cancelled by the desired correlator. Irrespective of high or low load, our proposed method can precisely estimate the interference. Furthermore, thermal noise, APD noise, and interference are included in the analysis. (C) 2001 John Wiley \& Sons, Inc. Microwave Opt Technol Lett 30: 1-3, 2001
\end{abstract}

Key words: optical CDMA; interference cancellation scheme

\section{INTRODUCTION}

A synchronous optical CDMA system can provide a greater number of simultaneous users than an asynchronous system [1]. However, in an optical CDMA system, multiple-access interference (MAI) will cause a penalty on system performance. To reduce the adverse impact of multiple-access interference, some alleviating methods such as using optical hard limiter(s) can effectively enhance system performance, but only in the case of a lightly loaded traffic situation.

Interference cancellation is an effective way to alleviate the adverse impact of multiple-access interference $[2,3,6]$. In [2], a simple scheme utilizes averaging interference cancellation to estimate multiple-access interference from the received optical signal. However, such a method can precisely estimate the interfering signals only in a high-load traffic situation.

Schemes using reference correlator(s) have been investigated to reduce the multiple-access interference $[3,6]$. In [6], some reference correlators are necessary in the interference cancellation schemes, thus reducing the cardinality of the signature sequence. To prevent a reduction of the cardinality of the signature sequence, all of the subscribers can use the same reference correlator based on a padded modified prime sequence [3], whereas using the reference correlator method will cause additional power splitting loss due to the optical tapped delay-line decoder. As in [3], we use the padding method so that the entire spreading sequence family has a unique cross-correlation value of "1." Combined with estimating the received optical signal and cancellation scheme, this approach can completely eliminate the multiple-access interference irrespective of high- or low-load traffic. Also, this scheme can cause an increase of about $3 \mathrm{~dB}$ power in sensitivity over that using the reference correlator in [3].

\section{SYSTEM DESCRIPTION}

The prime sequence [1], whose length is $p^{2}$, can be constructed from the modulo- $p$ multiplication of the Galois field $\operatorname{GF}(p)=\{0,1, \ldots, p-1\}$, where $p$ is a prime number. The padding subsequence to the signature sequence has been investigated to improve the cross-correlation property. For example, a prime code with padding $(p-1)$ zeros in each subblock of the codeword has been discussed [5].

Modified prime sequences are generated from time-shifted versions of the prime code, which can be applied to the synchronous systems [1]. The modified prime code has $p$ groups, and each contains $p$ mutually orthogonal code sequences. Each code sequence has $p^{2}$ chips (i.e., code length $=p^{2}$ ) with weight $p$. Sequences between any different groups have a cross-correlation value of " 1 ."

In [3], padding the same subblock of length $p$ to each group of the modified prime sequence can make the whole code family have a unique cross-correlation value of " 1. " Table 1 illustrates the padded modified prime sequences for $p=5$ [3]. This padded spreading code has the same cardinality as the original one, but the code weight and spreading length become $w=(p+1)$ and $F=p^{2}+p$, respectively.

The proposed interference cancellation scheme is shown in Figure 1. The received optical signal is split into two arms, with ratios $\alpha$ and $1-\alpha$, respectively. As the optical CDMA decoder employs optical tapped delay lines, this will cause a $1 /(p+1)$ power-splitting loss (in accordance with the code weight). We denote the desired signal as $S_{i}$ and the interfering signal as $S_{j}$. After parallel cancellation, the output $Z$ can be represented as

$$
\begin{aligned}
Z= & \frac{\alpha}{p+1}\left[S_{i} \cdot(p+1)+\sum_{j=1 ; j \neq i}^{N} S_{j}\right] \\
& -(1-\alpha)\left[S_{i} \cdot(p+1)+\sum_{j=1 ; j \neq i}^{N}(p+1) S_{j}\right]
\end{aligned}
$$

\begin{tabular}{|c|c|c|c|c|c|c|}
\hline Group & & & de Seque & nces & & \\
\hline 0 & $\begin{array}{l}C_{0,0}=10000 \\
C_{0,1}=00001 \\
C_{0,2}=00010 \\
C_{0,3}=00100 \\
C_{0,4}=01000\end{array}$ & $\begin{array}{l}10000 \\
00001 \\
00010 \\
00100 \\
01000\end{array}$ & $\begin{array}{l}10000 \\
00001 \\
00010 \\
00100 \\
01000\end{array}$ & $\begin{array}{l}10000 \\
00001 \\
00010 \\
00100 \\
01000\end{array}$ & $\begin{array}{l}10000 \\
00001 \\
00010 \\
00100 \\
01000\end{array}$ & $\begin{array}{l}10000 \\
10000 \\
10000 \\
10000 \\
10000\end{array}$ \\
\hline 1 & $\begin{array}{l}C_{1,0}=10000 \\
C_{1,1}=01000 \\
C_{1,2}=00100 \\
C_{1,3}=00010 \\
C_{1,4}=00001\end{array}$ & $\begin{array}{l}01000 \\
00100 \\
00010 \\
00001 \\
10000\end{array}$ & $\begin{array}{l}00100 \\
00010 \\
00001 \\
10000 \\
01000\end{array}$ & $\begin{array}{l}00010 \\
00001 \\
10000 \\
01000 \\
00100\end{array}$ & $\begin{array}{l}00001 \\
10000 \\
01000 \\
00100 \\
00010\end{array}$ & $\begin{array}{l}01000 \\
01000 \\
01000 \\
01000 \\
01000\end{array}$ \\
\hline 2 & $\begin{array}{l}C_{2,0}=10000 \\
C_{2,1}=00100 \\
C_{2,2}=00001 \\
C_{2,3}=01000 \\
C_{2,4}=00010\end{array}$ & $\begin{array}{l}00100 \\
00001 \\
01000 \\
00010 \\
10000\end{array}$ & $\begin{array}{l}00001 \\
01000 \\
00010 \\
10000 \\
00100\end{array}$ & $\begin{array}{l}01000 \\
00010 \\
10000 \\
00100 \\
00001\end{array}$ & $\begin{array}{l}00010 \\
10000 \\
00100 \\
00001 \\
01000\end{array}$ & $\begin{array}{l}00100 \\
00100 \\
00100 \\
00100 \\
00100\end{array}$ \\
\hline 3 & $\begin{array}{l}C_{3,0}=10000 \\
C_{3,1}=00010 \\
C_{3,2}=01000 \\
C_{3,3}=00001 \\
C_{3,4}=00100\end{array}$ & $\begin{array}{l}00010 \\
01000 \\
00001 \\
00100 \\
10000\end{array}$ & $\begin{array}{l}01000 \\
00001 \\
00100 \\
10000 \\
00010\end{array}$ & $\begin{array}{l}00001 \\
00100 \\
10000 \\
00010 \\
01000\end{array}$ & $\begin{array}{l}00100 \\
10000 \\
00010 \\
01000 \\
00001\end{array}$ & $\begin{array}{l}00010 \\
00010 \\
00010 \\
00010 \\
00010\end{array}$ \\
\hline 4 & $\begin{array}{l}C_{4,0}=10000 \\
C_{4,1}=00001 \\
C_{4,2}=00010 \\
C_{4,3}=00100 \\
C_{4,4}=01000\end{array}$ & $\begin{array}{l}00001 \\
00010 \\
00100 \\
01000 \\
10000\end{array}$ & $\begin{array}{l}00010 \\
00100 \\
01000 \\
10000 \\
00001\end{array}$ & $\begin{array}{l}00100 \\
01000 \\
10000 \\
00001 \\
00010\end{array}$ & $\begin{array}{l}01000 \\
10000 \\
00001 \\
00010 \\
00100\end{array}$ & $\begin{array}{c}00001 \\
00001 \\
00001 \\
00001 \\
00001 \\
\uparrow \\
\text { Padded } \\
\text { sequence }\end{array}$ \\
\hline
\end{tabular}

TABLE 1 Padded Modified Prime Sequence Code for $p=5$ 


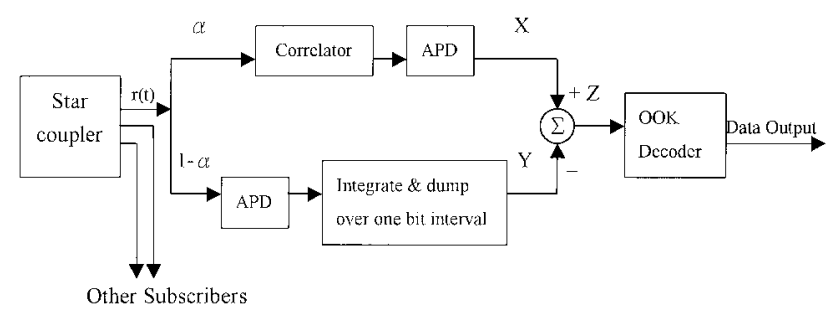

Figure 1 Receiver using interference cancellation scheme via integration over one bit interval $T_{b}$ from received optical signal

$$
\begin{aligned}
& =S_{i} \cdot(p+1)\left(\frac{\alpha}{p+1}-(1-\alpha)\right) \\
& \quad+\left[\frac{\alpha}{p+1}-(1-\alpha)(p+1)\right] \cdot \sum_{j=1 ; j \neq i}^{p^{2}-1} S_{j}
\end{aligned}
$$

where $N$ is the number of active users.

To suppress the interfering signals (i.e., to make the output signal irrelevant to interference), the value of $\alpha$ should be

$$
\alpha=\frac{(p+1)^{2}}{1+(p+1)^{2}}
$$

Remark: Without padding (i.e., only a modified prime sequence), the desired correlator cannot extract the impact of interfering signals from the same group (because of orthogonality). Hence, under low- to medium-load traffic, this scheme cannot operate effectively [2].

\section{SYSTEM PERFORMANCE ANALYSIS}

In this section, we take account of thermal noise, APD noise, and interference to analyze the system performance. The APD output can be modeled approximately as a Gaussian distribution [4]. The received optical signal intensity over a chip interval $T_{c}$ is modeled as a Poisson point process. The average number of absorbed photons is $\lambda_{s} T_{c}$, where $\lambda_{s}$ is the arrival rate of incident photons due to chip " 1 " transmission, which can be represented as $\lambda_{s}=\eta P_{w} / h f$. Here, $P_{w}$ is the optical power incident upon APD, $\eta$ is the APD quantum efficiency, $h$ is Planck's constant $\left(6.624 \times 10^{-34} \mathrm{~W} \cdot \mathrm{s}^{2}\right)$, and $f$ is the optical carrier frequency.

We use two Gaussian random variables $X$ and $Y$ to represent the APD outputs of the upper arm and lower arm, respectively, and their mean and variance are given by

$$
\begin{gathered}
\mu_{x}=G T_{c}\left[P_{w x} \cdot \lambda_{s}+I_{b} / e\right]+T_{c} \cdot I_{s} / e \\
\sigma_{x}^{2}=G^{2} F_{e} T_{c}\left[P_{w x} \cdot \lambda_{s}+I_{b} / e\right]+T_{c} \cdot I_{s} / e+\sigma_{\mathrm{th}}^{2} \\
\mu_{y}=G T_{c}\left[P_{w y} \cdot \lambda_{s}+F \cdot I_{b} / e\right]+F \cdot T_{c} \cdot I_{s} / e \\
\sigma_{y}^{2}=G^{2} F_{e} T_{c}\left[P_{w y} \cdot \lambda_{s}+F \cdot I_{b} / e\right]+F \cdot\left(T_{c} \cdot I_{s} / e+\sigma_{\mathrm{th}}^{2}\right)
\end{gathered}
$$

where $G$ is the average APD gain, $I_{s}$ is the APD surface leakage current, $I_{b}$ is the APD bulk leakage current, $e$ is the electron charge, $P_{w x}$ and $P_{w y}$ are the optical power incident upon the APD in the upper and lower arms, respectively, and $F_{e}$ is the excess noise factor given by

$$
F_{e}=K_{\mathrm{eff}} G+(2-1 / G)\left(1-K_{\mathrm{eff}}\right)
$$

where $K_{\text {eff }}$ is the APD effective ionization ratio and $\sigma_{\text {th }}^{2}$ is the variance of thermal noise, given by

$$
\sigma_{\text {th }}^{2}=2 K_{B} T_{r} T_{c} /\left(e^{2} R_{L}\right)
$$

where $T_{r}$ is the receiver noise temperature, $K_{B}$ is Boltzmann's constant and $R_{L}$ is the receiver load resistance.

After cancellation, the decision variable $Z=X-Y$ is also Gaussian, and since $X$ and $Y$ are independent, its mean and variance are given by

$$
\begin{gathered}
\mu_{z}=\mu_{x}-\mu_{y} \\
\sigma_{z}^{2}=\sigma_{x}^{2}+\sigma_{y}^{2} .
\end{gathered}
$$

Assuming that transmitted data are equally likely, the conditional probability density function of $k$ users transmitting bit " 0 " or " 1 " is $\operatorname{Pr}[k / N-1]=\left(\begin{array}{c}N-1 \\ k\end{array}\right) \cdot 2^{-(N-1)}$.

Therefore, the bit-error probability can be represented as

$$
\begin{aligned}
P_{e}= & \frac{1}{2}\left[\operatorname{Pr}\left(Z>\theta / S_{i}=0, N-1\right)\right. \\
& \left.+\operatorname{Pr}\left(Z \leq \theta / S_{i}=1, N-1\right)\right] \\
= & \frac{1}{2} \sum_{k=0}^{N-1}\left\{\operatorname{Pr}\left(Z>\theta / S_{i}=0, k, N-1\right)\right. \\
& \left.+\operatorname{Pr}\left(Z \leq \theta / S_{i}=1, k, N-1\right) \cdot\right\} \operatorname{Pr}[k / N-1]
\end{aligned}
$$

where $\theta$ is the OOK decision threshold, which is usually set to be half of the mean of the decision variable $Z$ [3].

\section{NUMERICAL RESULT AND DISCUSSION}

The numerical parameters used here are the same as in [3, 4]. Figure 2 shows the bit-error probability comparisons for the reference correlator (Ref-Cor) [3] and the proposed interference estimation (IE) scheme with full-load subscribers $\left(p^{2}\right)$ for $p=5,7$, and 11 , respectively. In the present scheme, the performance can be improved by about $3 \mathrm{~dB}$ in sensitivity over that using the reference correlator [3] because using the reference correlator in the lower arm of the interference canceller will lead to a power-splitting loss $(1 / p+1)$ caused by the optical tapped delay-line decoder. To obtain a larger

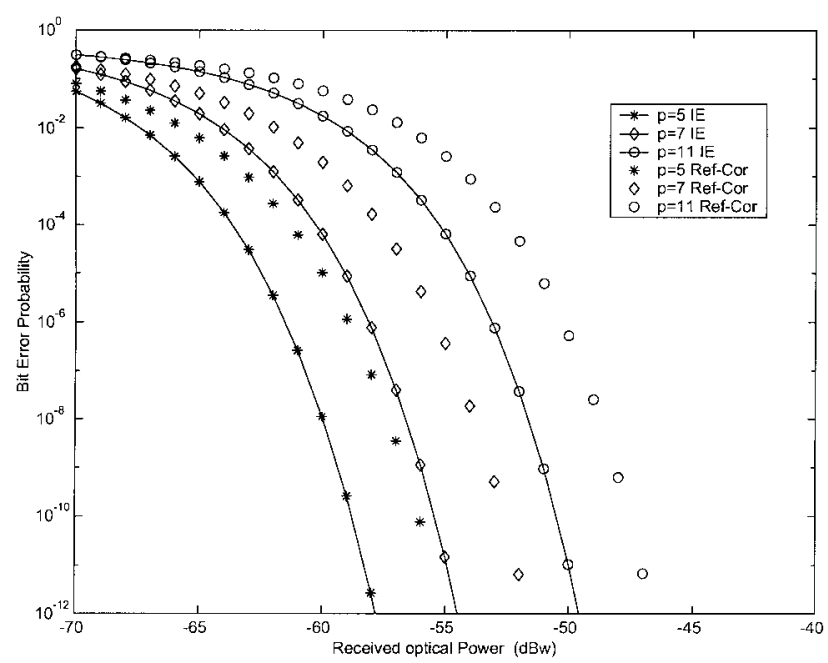

Figure 2 Bit-error probability comparisons for reference correlator [3] and interference estimation scheme with full-load subscribers for $p=5,7$, and 11 , respectively 
aggregate capacity, increasing the optical power and using a larger prime number $p$ are the necessary costs.

Due to the unique cross-correlation value of the padded modified prime sequence, our proposed scheme can precisely estimate the multiple-access interference from the received optical signal. Consequently, via parallel cancellation, the desired signal can be exactly detected.

\section{REFERENCES}

1. W.C. Kwong, P.A. Perrier, and P.R. Prucnal, Performance comparison of asynchronous and synchronous code division multiple access techniques for fiber-optic local area networks, IEEE Trans Commun 39 (1991), 1625-1634.

2. H. Walle and U. Killat, Averaging interference cancellation for optical DS-CDMA systems, Proc IEEE 4th Int Symp Spread Spectrum Techniques and Appl, 1996, vol. 2, pp. 609-613.

3. M.Y. Liu and H.W. Tsao, Cochannel interference cancellation via employing a reference correlator for synchronous optical CDMA systems, Microwave Opt Technol Lett 25 (2000).

4. H.M. Kwon, Optical orthogonal code division multiple access system-Part I: APD noise and thermal noise, IEEE Trans Commun 42 (1994), 2470-2479.

5. G.C. Yang and W.C. Kwong, Prime codes, Electron Lett 31 (1995).

6. H.M.M. Shalaby, Synchronous fiber-optic CDMA systems with interference estimators, J Lightwave Technol 17 (1999), 2268-2275.

(c) 2001 John Wiley \& Sons, Inc.

\section{MINIATURIZED $20 \mathrm{GHz}$ CPW QUADRATURE COUPLER USING CAPACITIVE LOADING}

\author{
R. Baliram Singh ${ }^{1}$ and T. M. Weller ${ }^{1}$ \\ ${ }^{1}$ Department of Electrical Engineering \\ University of South Florida \\ Tampa, Florida 33620
}

\section{Received 21 January 2001}

\begin{abstract}
This paper describes coplanar waveguide (CPW) quadrature couplers that utilize metal-insulator-metal (MIM) capacitors at each corner, eliminating the need for air bridges and significantly reducing the coupler footprint. A $20 \mathrm{GHz}$ coupler on high-resistivity silicon with branch lengths of $40^{\circ}(660 \mu \mathrm{m})$ is presented-the area is $80 \%$ smaller than a conventional design. A measured insertion loss of $\sim 4.5$ $\pm 0.5 \mathrm{~dB}$ was achieved at $20 \mathrm{GHz}$. (C) $2001 \mathrm{John}$ Wiley \& Sons, Inc. Microwave Opt Technol Lett 30: 3-5, 2001.
\end{abstract}

Key words: coplanar waveguide; quadrature coupler; capacitive loading; thin-film capacitor

\section{INTRODUCTION}

Directional couplers are passive microwave components that may be used for power division and/or combining in microwave circuits such as mixers, balanced amplifiers, data modulators, phase shifters, and feed networks in antenna arrays. Conventional quadrature hybrid couplers utilize $\lambda / 4$ $\left(90^{\circ}\right)$ transmission lines. However, as demonstrated in [1], the lengths of the $\lambda / 4$ lines can be reduced by increasing the characteristic impedance and introducing lumped capacitance at the ends of the lines. Additional examples of capacitive loading in coupler design can be found in [2-3].

Contract grant sponsor: Raytheon C3 Systems, St. Petersburg, FL
In this paper, miniaturized CPW quadrature couplers using thin-film MIM capacitors are described. The work is distinguished from prior publications in that a CPW coupler is designed using three capacitors located at each corner in place of the conventional air bridges, thus providing ground equalization along with size reduction. Previous works were done mostly on microstrip, while those done with the CPW topology included only one capacitor located at each corner, and still utilized air bridges. New guidelines for coupler design are also given herein.

A summary of the design approach is presented in the following section. The analysis provides for the determination of necessary characteristic impedance and capacitive loading for a given electrical length of the coupler branch arms. The simulation and optimization of the couplers are presented in Section III, followed by experimental results in Section IV. The designs studied here used $\sim 1-\mu$ m-thick $\mathrm{Cr}-\mathrm{Ag}-\mathrm{Cr}-\mathrm{Au}$ layers for the MIM and CPW metal, and a $0.58-\mu$ m-thick SiO layer $\left(\varepsilon_{r}=6\right)$ for the capacitor dielectric [4].

\section{DESIGN APPROACH}

The geometry parameters associated with a capacitively loaded hybrid coupler can be simplified as shown in Figure 1. To minimize the number of variables, the down and across branches are taken to be equal in length $\left(\theta_{1}=\theta_{2}=\theta\right)$, and the capacitance at each corner is also assumed to be equal. Thus, there are four quantities to be determined: the electrical length $(\theta)$, the impedance of the down and across branches $\left(Z_{1}, Z_{2}\right)$, and the capacitance (Cap).

The approach taken here is to solve for $Z_{1}, Z_{2}$, and Cap as a function of $\theta$ such that the even- and odd-mode conditions for a conventional quadrature hybrid are satisfied [5]. For both the even and odd modes, we want

$$
S_{11}=0 \text {. }
$$

For the even mode, we require

$$
S_{21}=\frac{-(1+j)}{\sqrt{2}}
$$

and for the odd mode,

$$
S_{21}=\frac{(1-j)}{\sqrt{2}} \text {. }
$$

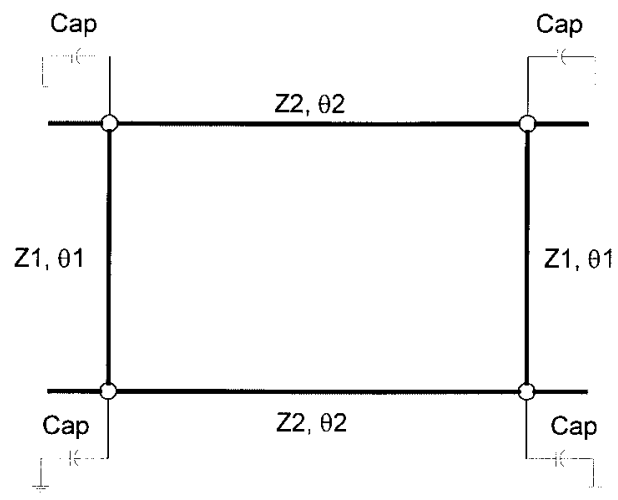

Figure 1 Geometry parameters associated with the $90^{\circ}$ hybrid coupler 